For reprint orders, please contact reprints@future-drugs.com

\title{
Progress in vaccination against cancer (PIVAC) 2002
}

\author{
July 18-20, 2002, Nottingham, UK \\ Geng Li and Robert C Rees'

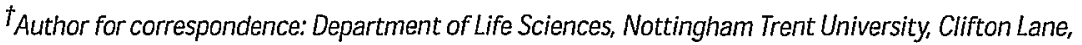 \\ Clifton, Nottingham NG17 8NS, UK, Tel.:+441158486342, Fax:+447158486636, robert.rees@ntu.ac.uk
}

Expert Rev. Vaccines 1(3), 261-263 (2002)

Progress in Vaccination Against Cancer (PIVAC) 2002 was held in Nottingham, UK, July 18-20, 2002. Over 100 delegates from 17 countries attended this scientific event. The following review summarizes the main issues arising from the presentations at the conference.

\section{Identification \& characterization of tumor antigens}

The identification of immunogenic molecules which are overexpressed in malignant tissues, but not in essential normal cells, such as brain, lung, heart, liver and kidney, is crucial for the development of cancer vaccines. The serological identification of tumor antigens by a recombinant expression cloning (SEREX) approach was discussed during the meeting. D Schadendorf, Heidelberg, Germany, reported that they had identified several antigens by immunoscreening of cDNA libraries constructed from normal testis and cutaneous lymphomas with sera from patients with cutaneous lymphoma. Some of these antigens were only expressed in testis and tumor tissues and also reacted with sera from patients with cutaneous lymphoma [1]. A Miles, Nottingham, UK, applied a similar approach to identify prostatic carcinoma antigens and isolated three antigens which were overexpressed in normal testes and cancerous tissues, as determined by real-time reverse transcriptase polymerase chain reaction (RT-PCR). SEREX-defined antigens were also found in acute myeloid leukemia (B Guinn, London, UK) [2].
Application of mass spectrometric analytical techniques in conjunction with Ciphergen protein chip technology, coupled with trainable artificial neural network programs, may allow the identification of validated biomarkers correlated with disease progression, treatment success or tumor escape. These biomarkers warrant further characterization to assess their suitability as potential therapeutic targets ( $S$ Mian, Nottingham, UK, J Tolson, Tubingen, Germany) [3].

The wide expression of the human telomerase catalytic subunit (hTERT) in tumors makes it an interesting universal candidate antigen for cancer immunotherapy. G Gaudernack, Oslo, Norway, has used several new epitopes of hTERT that can elicit both CD4 and CD8 cell responses against hTERT in a clinical Phase I trial in patients with advanced pancreatic cancer, non-small cell lung cancer and melanoma. No serious adverse effects related to the treatment have been observed and $75 \%$ of patients who received high-dose vaccine demonstrated an immune response measured either by delayed-type hypersensitivity (DTH) reaction or in vitro tests. Furthermore, a survival advantage was observed for patients receiving the high-dose vaccine compared with those receiving the lower dose [4].

Proteins encoded by $b c r / a b l$ fusion genes generated by $t(9 ; 22)$ translocation in chronic myelogenous leukemia (CML) patients are not expressed in normal cells. $G$ Pawelec, Tubingen,
Germany, described synthetic peptides representing sequences encompassing the break point region of both $\mathrm{bcr} / \mathrm{abl}$ and $a b 1 / b c r$ that were immunogenic for both CD4 and CD8 cells in vitro. IA Dodi, London, UK, reported on CMLspecific peptides eluted from human leukocyte antigen (HLA)-A3 or HLAB8 K652 (CML cell line) transfectants as well as from CML cells isolated from HLA-A3 patients. Tetramers of HLAA3 and HLA-B8 with the corresponding eluted peptides were produced and used to detect antigen-specific cytotoxic T-lymphocytes (CTLs) from HLA-A3 and HLA-B8 patients at various stages of the disease and treatment [5].

A combination of vaccines targeting both tumor endothelial and epithelial cells may lead to vascular collapse and increase extravasation leading to tumor regression. Tie- 2 is a receptor tyrosine kinase overexpressed in tumor endothelial cells. Both CD4 and CD8 epitopes within Tie-2 were identified. CD8 epitopes can stimulate CTL responses in HLA-A2 transgenic mice and kill endothelial cells overexpressing Tie-2. CD4 epitopes can stimulate a Th1 response in vitro. CD55 is overexpressed in tumor cells and a human anti-idiotypic antibody, 105AD7, that mimics the CD55 antigen has been produced. Colorectal tumor patients immunized with 105AD7 showed Tcell blastogenesis against tumor cells expressing CD55 and a significant increase in tumor cell apoptosis (LG Durrant, Nottingham, UK) [6].

DE Speiser, Lausanne, Switzerland, has vaccinated melanoma patients with Melan-A/MART-1 peptides. The vaccines were well tolerated. One of 21 patients showed disease stabilization and two out of 21 patients who had the strongest activation and expansion of tumor antigen-specific T-cells experienced regression of skin, lymph nodes and lung metastases [7]. JM Rojas, Nottingham, UK, reported that major histocompatibility complex (MHC) class II 
epitopes derived from p53 and gp100 were immunogenic in a transgenic mouse model and demonstrated that HLA-DR transgenic mice represent a suitable model for the discovery of $\mathrm{MH}-\mathrm{IC}$ class II-restricted epitopes derived from tumor antigens.

\section{Dendritic cell-based vaccination}

Dendritic cells (DCs) play a central role in the immune system. Without optimal DC antigen-presenting function there can be no antitumor immunity. However, it is important to note that $\mathrm{DCs}$ that present the relevant $\mathrm{MHC}$-peptide complexes qualify as potential targets and are at risk of being eliminated by the CTLs they have activated. Immature DCs are indeed susceptible to CTLinduced killing, but the mature $D C s$ activated by Anti-CD40 or lipopolysaccharide (LPS) are resistant to this effect. Protection is achieved by expression of a serine protease inhibitor (SPI) -6 and its human homolog PI-9 induced by AntiCD40 and LPS (R Offringa, Leiden, The Netherlands) [8].

AM Jackson, Leeds, UK, described recombinant mycobacteria expressing tumor antigens able to infect and cause marked maturation of $\mathrm{DCs}$, resulting in cytokine secretion and increased T-cell stimulatory capacity. E Kaminski, Plymouth, UK, reported that autologous Tcells treated with interleukin (IL)-15 prior to culture with tumor lysate-pulsed $D C_{s}$, exhibited a significant increase in $\mathrm{T}$-cell proliferation, interferon (IFN) $-\gamma$ secretion and specific cytotoxicity to target cells. HS Pandha, London, UK, has developed a method of culturing then cryopreserving, monocyte-derived DCs after pulsing with irradiated allogenic prostatic cancer cell lysate. This process increased DC maturation and thus enhanced their antigen-presenting capability. In the study, 14 patients with metastatic prostatic cancer received the DC vaccines (a Phase I/II clinical trial) and four experienced a PSA response: two with an arrest in PSA levels and two showing reduction in PSA levels.

V Cerundolo, Oxford, UK, discussed the strategies that could generate a broad and long-lasting antitumor-specific immunity. They engineered a string of HLA-A2 and -A1-restricted melanoma epitopes which were cloned into naked plasmid DNA, vaccinia virus, modified vaccinia Ankara virus (MVA) and Semliki forest virus (SFV) and vaccinated HLA-A2 transgenic mice with different combinations of the polyepitope constructs. Their results demonstrated that competition for antigen recognition at the surface of antigen-presenting cells (APCs) may significantly skew the immune response and this competition needs to be taken into account for the development of vaccination strategies to optimally induce polyvalent CTL response. They identified two alternative protocols for inducing polyvalent CTL response, based on the injection of either increasing numbers of APCs infected with vaccinia virus encoding the polyepitope construct, or a mixture of viruses each coding a separate antigen [9].

P Straten, Copenhagen, Denmark, described TCR clonotype mapping utilizing RT-PCR and denaturing gradient gel electrophoresis (DGGE). Using these sensitive assays it was demonstrated that predominant T-cell clones infiltrating melanoma tissue do not reflect T-cell populations expanded in vitro from the same tissue. This questions the interpretation and potential importance of data obtained on cultured TILs. However, the application of DGGE has shown a correlation between circulating T-cell clones and TILs during IL-2 therapy.

Tumor escape from immune surveillance Tumor cells develop various strategies to escape immune surveillance and effector cells also express negative regulatory receptors which provides further examples of the double-edged sword of immunotherapy. Finding ways of preventing tumor escape by multiple strategies will be the main challenge for immunotherapy of cancer. $\mathrm{KJ}$ Maimberg, Stockholm, Sweden, described how hydrogen peroxide $\left(\mathrm{H}_{2} \mathrm{O}_{2}\right)$, released by tumor infiltrating macrophages, can impair the cytokine production and function of T- and natural killer (NK) cells. Exogenous supplied antioxidants, such as high doses of dietary vitamin $\mathrm{E}$, may protect the immune cells from the attack of
$\mathrm{H}_{2} \mathrm{O}_{2}$ by increasing CD4/CD8 ratio and IL-2 production. He also pointed out that IFN- $\gamma$ treatment of ovarian carcinoma cells protected them from lysis by CTL. This phenomenon was dependent on the enhanced signaling via CD94/NKG2A receptors expressed on the T-cells [10]. On the other hand, IFN- $\gamma$ is essential for tumor resistance in animal models including the Stat-6 knockout model described by $S$ Ostrand-Rosenberg, Baltimore, USA [11].

M Ahmad, Nottingham, UK, reported that direct intratumor injection of disabled herpes simplex viruses, DISC/HSV/mGM-CSF, into established tumors induced tumor rejection in $40-60 \%$ of treated animals (regressor), while the remaining mice had progressive tumors (progressor). To identify the mechanisms responsible for the failure of immunotherapy, they examined whether the APCs of progressor mice were functional and induced a CTL response - they reported that the APCs from the progressor mice did not support CTL generation. They suggest that either the presence of suppressor elements in the progressor paranchymal APC fraction or the APCs from progressor mice do not effectively present antigens to CTLs [12]. As mentioned in the section on DC-based vaccination, DCs protect themselves against lysis by the CTLs they induced by expressing PI-9/SPI-6. Unfortunately, a variety of human and murine tumor cells also express PI-9/ SPI-6, resulting in the resistance of tumor cells to CTL-mediated killing.

Loss or downregulation of TAP expression prevents presentation of the tumor antigens on the surface of tumor cells and therefore allows them to avoid recognition by CTLs. WA Jefferies, Vancouver, Canada, described how transfection of mouse melanoma B16F10 (TAPdeficient) cells with vaccinia vector carrying TAP1 could enhance tumor-specific T-cell generation and reduce tumor burden in mice. Inclusion of TAP in cancer therapy might provide a general method for increasing immune responses against tumors regardless of the antigenic complement of tumor or the $\mathrm{MHC}$ haptotypes of the host [13]. 


\section{Acknowledgements}

We acknowledge support from The British Society for Immunology. Special thanks are extended to Sandra Lowne, Ben Stanley (BSI) and Glenda Kill (Nottingham Trent University, UK) for their

\section{References}

1 Eichmuller S, Usener D, Dummer R, Stein A, Thiel D, Schadendorf D. Serological detection of cutaneous T-cell lymphomaassociated antigens. Proc. NatI Acad. Sci. USA 98, 629-634 (2001).

2 Guinn B-A, Collin F, Li G, ReesRC, Mufti GJ. Optimised SEREX technique for the identification of leukemiaassociated antigens. J. Immunol. Methods 264, 207-214 (2002).

3 Ball G, Mian S, Holding F et al. An integrated approach utilizing artificial neural networks and SELDI mass spectrometry for the classification of human tumours and rapid identification of potential biomarkers. Bioinformatics 18 , 395-404 (2002).

4 Saeboe-Larssen S, Fossberg E, Gaudernack G. mRNA-based electrotransfection of human dendritic cells and induction of cytotoxic T-lymphocyte responses against the telomerase catalytic subunit (hTERT). J. Immunol. Methods 259, 191-203 (2002).

5 Clark RE, Dodi IA, Hill SC et al. Direct evidence that leukemic cells present HLAassociated immunogenic peptides derived

help in organizing the meeting. We wish to acknowledge support by grants from The Cancer and Polio Research Fund and The John and Lucille van Geest Foundation.

from the BCR-ABL b3a2 fusion protein. Blood 98, 2887-2893 (2001).

6 Durrant LG, Parsons T, Moss R, Spendlove I. Carter G, Carr F. Human anti-idiotypic antibodies can be good immunogens as they target FC receptors on antigenpresenting cells allowing efficient stimulation of both helper and cytotoxic Tcell responses. Int. J. Cancer 92, 414-420 (2001).

7 Pittet MJ, Zippelius A, Valmori D, Speiser DE, Cerottini JC, Romero P. Melan-A/ MART-1-specific CD8 T-cells: from thymus to tumor. Trends Immunol. 23 , 325-328 (2002).

8 Medema JP, Schuurhuis DH, Rea D et al. Expression of the serpin serine protease inhibitor 6 protects dendritic cells from cytotoxic T-lymphocyte-induced apoptosis: differential modulation by T-helper Type 1 and Type 2 cells. J. Exp. Med. 194, 657667 (2001).

9 Palmowski MJ, Choi EM, Hermans IF et al. Competition between CTL narrows the immune response induced by primeboost vaccination protocols. I. Immunol. 168, 4391-4398 (2002).
10 Malmberg KJ, Arulampalam V, Ichihara F et al. Inhibition of activated/memory (CD45RO(t)) T-cells by oxidative stress associated with block of NF-kappaB activation. J. Immunol. 167, 2595-2601 (2001).

11 Ostrand-Rosenberg S, Grusby MJ, Clements VK. Cutting edge: STAT6deficient mice have enhanced tumor immunity to primary and metastatic mammary carcinoma. J. Immunol. 165, 6015-6019 (2000).

12 Ali SA, Lynam J, McLean CS et al. Tumor regression induced by intratumor therapy with a disabled infectious single cycle (DISC) herpes simplex virus (HSV) vector, DISC/HSV/murine granulocytemacrophage colony-stimulating factor, correlates with antigen-specific adaptive immunity. J. Immunol. 168, 3512-3519 (2002).

13 Alimonti J, Zhang QJ, Gabathuler R, Reid $G$, Chen SS, Jefferies WA. TAP expression provides a general method for improving the recognition of malignant cells in vivo. Nat. Biotechnol. 18, 515-520 (2000). 